\title{
Beam commissioning in the first superconducting segment of the Facility for Rare Isotope Beams
}

\author{
P. N. Ostroumov, T. Maruta, S. Cogan, K. Fukushima, S. H. Kim, S. Lidia, F. Marti, A. S. Plastun, \\ J. Wei, T. Yoshimoto, T. Zhang, and Q. Zhao \\ Facility for Rare Isotope Beams (FRIB), Michigan State University, East Lansing, Michigan 48824, USA
}

(Received 16 June 2019; published 7 August 2019)

\begin{abstract}
Linac segment 1 (LS1) of the FRIB driver linac is composed of 15 cryomodules, consisting of 104 superconducting (SC) resonators and $39 \mathrm{SC}$ solenoids. Four ion beam species ( $\mathrm{Ne}, \mathrm{Ar}, \mathrm{Kr}$, and $\mathrm{Xe}$ ) were successfully accelerated up to $20.3 \mathrm{MeV} / \mathrm{u}$ in LS1 and transported to the designated beam dumps located in folding segment 1 (FS1). 100\% beam transmission was measured through all cryomodules and the warm section of LS1. High-power equivalent beams were delivered to the beam dump in two modes: pulsed and continuous wave (cw). In the pulsed mode, the peak intensity of the argon beam was $14.8 \mathrm{p} \mu \mathrm{A}$ at $3 \%$ duty factor, which constitutes $30 \%$ of the FRIB design intensity for this particular ion beam. A cw argon beam was accelerated, demonstrating that the FRIB linac in its current configuration is the highest-energy $\mathrm{cw}$ superconducting hadron linac in the world. This paper presents a detailed study of beam dynamics in LS1 prior to and after charge stripping with a carbon foil.
\end{abstract}

DOI: 10.1103/PhysRevAccelBeams.22.080101

\section{INTRODUCTION}

In February 2019, the FRIB driver accelerator became the highest-energy continuous wave hadron linac in the world. This significant milestone was achieved after the acceleration of four ion species $\left({ }^{40} \mathrm{Ar}^{9+},{ }^{86} \mathrm{Kr}^{17+},{ }^{10} \mathrm{Ne}^{6+}\right.$, and ${ }^{129} \mathrm{Xe}^{26+}$ ) to $20.3 \mathrm{MeV} / \mathrm{u}$ in 14 cryomodules with 99 of two types of superconducting (SC) cavities: $\beta_{\text {opt }}=0.041$ and $\beta_{\text {opt }}=0.085$. Three one-week beam shifts were scheduled during February through April 2019 and alternated with the ongoing equipment installation in the tunnel. After completion of the tuning and setting all accelerator parameters, the following beam studies were performed: (i) measurement of transverse beam parameters, beam matching, and optimization of beam envelopes; (ii) measurement of the charge state distribution of all four ion species after stripping with thin carbon foils; (iii) study of beam loading compensation; (iv) acceleration of a high-power equivalent beam; (v) transverse beam emittance measurements after the stripping, the effect of foil thickness and beam size on the stripper on emittance growth; and (vi) demonstration of the capability of linac segment 1 (LS1) to accelerate dual charge state beams simultaneously.

This paper consists of three sections describing (i) the acceleration of four ion species in several different modes,

Published by the American Physical Society under the terms of the Creative Commons Attribution 4.0 International license. Further distribution of this work must maintain attribution to the author(s) and the published article's title, journal citation, and DOI. including a high peak current mode, (ii) the study of beam properties after the stripping with a carbon foil, and (iii) an empirical demonstration of LS1 capability to accelerate dual charge state heavy ion beams.

\section{ACCELERATION OF HEAVY ION BEAMS}

The FRIB driver linac layout is shown in Fig. 1. Ion beams were accelerated in the shaded fraction of LS1 and folding segment 1 (FS1), which includes 14 accelerating cryomodules, a bunching cryomodule, a set of carbon foils for stripping, a room-temperature IH-type buncher, and two beam dumps (FS1a and FS1b) [1]. Prior to the beam commissioning, all SC cavities were cooled down to $4.5 \mathrm{~K}$ and conditioned at accelerating gradients exceeding the design value by $10 \%$. $\beta_{\text {opt }}=0.041$ cavities provide $0.81 \mathrm{MV}$, and $\beta_{\text {opt }}=0.085$ cavities provide $1.78 \mathrm{MV}$ design accelerating voltages for the beam entering the cavities with the optimal velocity. The cold section of the linac contains 39 SC solenoids, which were set to provide $\sim 60^{\circ}$ phase advance of transverse betatron oscillations in each focusing period. Because of the irregular focusing period length, the exact values of phase advances are slightly different as follows from the computer simulations with the FLAME code [2] (see Fig. 2). Working with a very low beam power, less than $2 \mathrm{~W}$, a phase scan procedure was applied to all $102 \mathrm{SC}$ cavities available for operation at design accelerating fields to accelerate the ${ }^{40} \mathrm{Ar}^{9+}$ beam to $20.3 \mathrm{MeV} / \mathrm{u}$ [3]. The planned liquid lithium stripper is not yet available, so a carbon foil wheel was used as a charge stripper. The beam absolute energy was measured after each cavity using a pair 


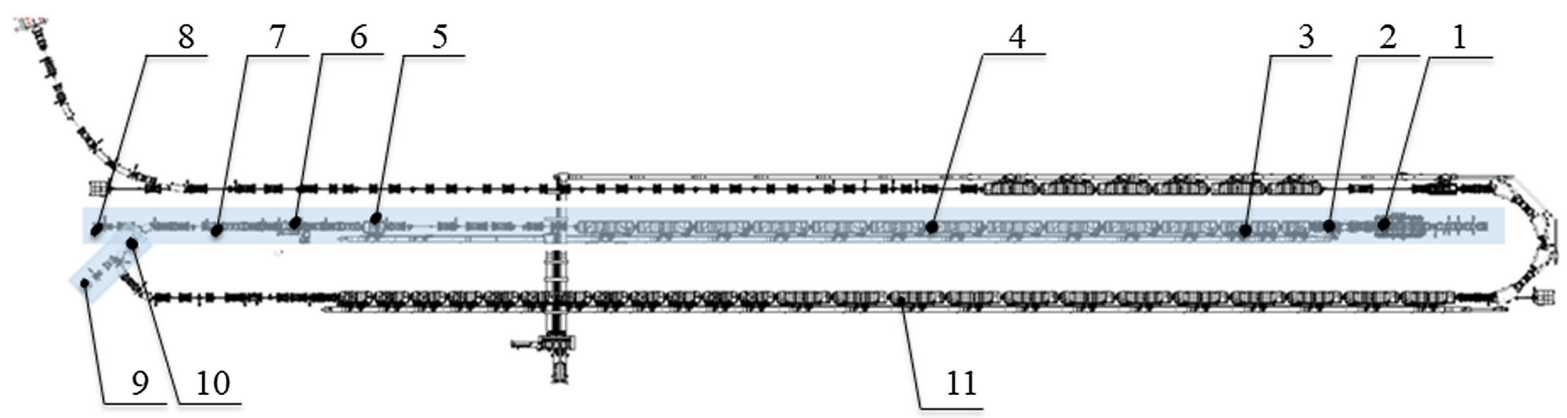

FIG. 1. FRIB layout. (1) Radio frequency quadrupole (RFQ), (2) medium energy beam transport (MEBT), (3) the first three cryomodules with $12 \beta_{\text {opt }}=0.041$ SC cavities, (4) 11 cryomodules with $88 \beta_{\text {opt }}=0.085$ SC cavities, (5) bunching cryomodule with four $\beta_{\text {opt }}=0.085 \mathrm{SC}$ cavities, (6) charge stripper, (7) normal conducting (NC) buncher, (8) beam dump FS1a, (9) beam dump FS1b, (10) charge selecting slits, (11) linac segment 2 (LS2).

of beam position monitors (BPMs) located downstream of the cavity being tuned. Typical beam parameters during the phase scan were a pulse length of $50 \mu \mathrm{s}$, a repetition rate of $5 \mathrm{~Hz}$, and a peak current of several $\mu \mathrm{A}$. Two SC cavities, one in the accelerating section and one in the matching cryomodule, were not available for the beam acceleration at this time due to a frequency tuner issue.

A large variety of beam diagnostics devices were available during the beam commissioning in LS1 and FS1 including the following. (i) Seven ac-coupled beam current monitors (BCMs) including two BCMs installed immediately upstream and downstream of the RFQ.-An electrostatic chopper located in the low energy beam transport was used to produce a pulsed beam structure necessary for BCM beam current measurements and control the average beam power. (ii) $70 \mathrm{BPMs}$.- In addition to the beam position, the BPMs provide a beam-induced phase. Any two BPMs can be paired for the absolute energy measurements. (iii) 14 halo monitor rings (HMRs).- The HMR is an isolated niobium ring with a beam aperture of 30 and $33 \mathrm{~mm}$ in the sections of $\beta_{\mathrm{opt}}=0.041$ and $\beta_{\mathrm{opt}}=0.085$ cavities, respectively, while the physical aperture of all LS1 cavities is $36 \mathrm{~mm}$. They are installed in the warm sections after each cryomodule. During operation, HMRs are biased at $100 \mathrm{~V}$ to suppress secondary

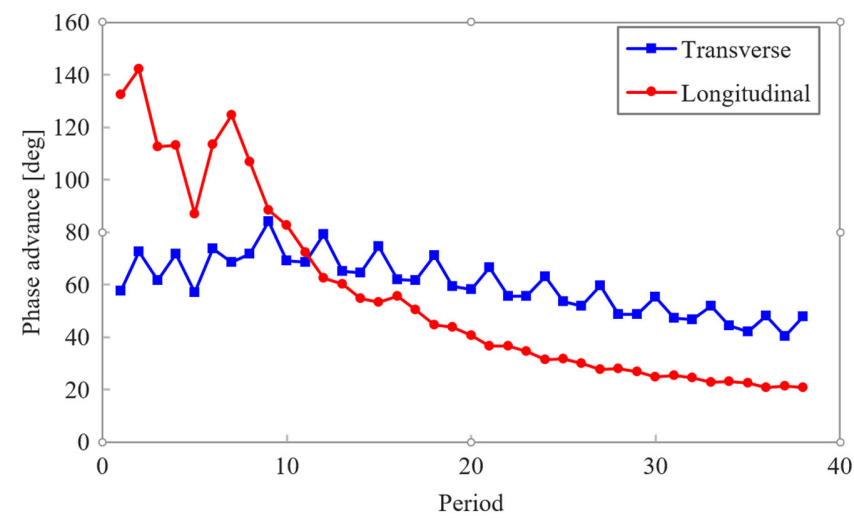

FIG. 2. Transverse and longitudinal phase advances along the LS1 accelerator section. electrons. (iv) Four profile monitors (PMs).-The profile monitor devices consist of actuated horizontal, vertical, and $45^{\circ}$-angled wires. One PM is located in the MEBT, two PMs are located between the cold section and the stripper, and one more PM is located upstream of the FS1 a beam dump. (v) 11 scintillator-based neutron monitors spaced along the LS1 cryomodules. (vi) Eight parallel plate ion chambers in the FS1 section, situated around the carbon charge stripper and the beam dumps.

One of the beam commissioning tasks is the transverse beam dynamics verification in LS1 and FS1. The transverse beam dynamics was verified by beam profile measurements, evaluation of the beam Twiss parameters, and root mean squared (rms) emittances. Two methods were utilized to evaluate the beam Twiss parameters and rms emittances in the horizontal and vertical phase space planes: (a) beam profile measurements in three locations along the beam line and (b) beam profile measurements in a single location while an upstream quadrupole is varied (the quadrupole scan method). The second method was primarily applied to measure the beam parameters after the stripping foil, since only one profile monitor is available in this section of the linac. During the quadrupole scan, a significant beam mismatch develops with the following section of the beam transport system. To avoid beam losses during the scans, a procedure was developed that includes an optimization of quadrupole fields in downstream quadrupoles to restore the beam matching. Beam rms envelopes during a typical quadrupole scan procedure calculated by the FLAME code [2] are shown in Fig. 3. In this example, four quadrupoles downstream of the profile monitor were used to restore beam matching with the following beam transport system. No beam losses were observed during these measurements.

The beam transport section after the cold linac section serves several functions: (a) to form a small beam size in the $\mathrm{SC}$ and normal conducting (NC) matching resonators; (b) to form a very small round beam with $0.5 \mathrm{~mm}$ rms radius on the charge stripper, and (c) to provide no-loss beam transmission to the beam dumps. Figure 4 presents the rms beam envelopes in the beam transport section from 


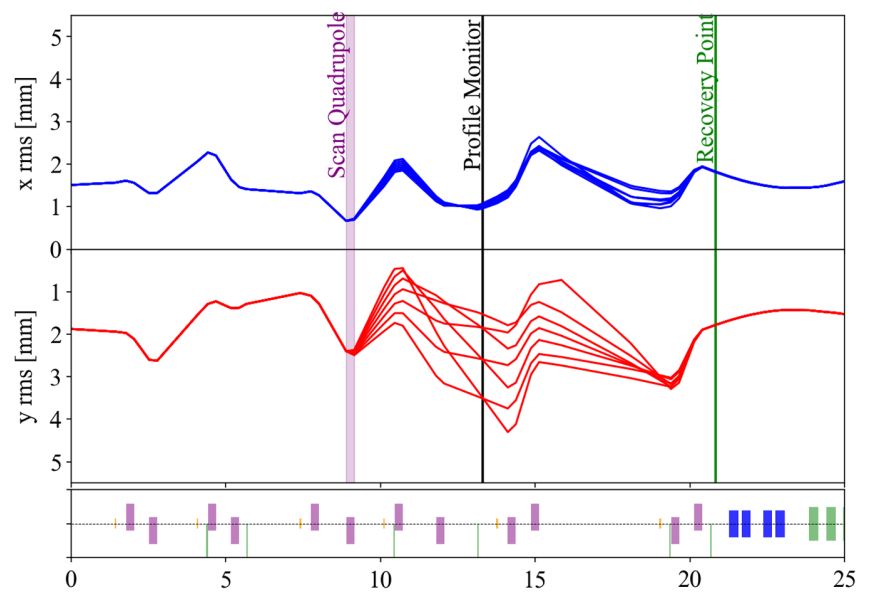

FIG. 3. Argon beam rms envelopes during a quadrupole scan.

the last cryomodule to the beam dump FS1a based on Twiss parameter evaluation using method (a) discussed above. The beam in the transport section was tuned in order to minimize the rms envelope size, as seen in Fig. 4. The transverse beam optics is consistent with the design, while achieving a minimal emittance growth of approximately $10 \%$, as listed in Table I. The emittance is independent from the beam peak current due to negligible space charge effects, as expected.

\section{A. Beam steering correction}

The $\mathrm{x} / \mathrm{y}$ transverse motion in the linac is strongly coupled due to the focusing with SC solenoids. The misalignments of both focusing elements and accelerating cavities result in beam centroid offsets along the linac. In addition, there is a contribution to beam steering due to the dipole component in the electromagnetic field of quarter wave resonators [4]. Therefore, some coherent oscillations of the beam central trajectory in LS1 are expected.
Combined SC magnets located inside the cryomodules include focusing solenoids and dipole magnets for beam steering corrections [5,6]. There are $69 \mathrm{BPMs}$ and 56 twoplane steering magnets between the RFQ and FS1a beam dump. Several techniques can be used for the correction of the beam central trajectory. The optics response matrix (ORM) method [7] was developed and applied for such a correction. The ORM-based algorithm was implemented in the FRIB control network as a dedicated GUI application to perform the trajectory correction in an efficient, convenient, and user-friendly way. The ORM algorithm is implemented as follows. Let us assume that there is a section of the accelerator with $m$ BPMs and $n$ dipole magnets. The ORM method is based on the evaluation of a response matrix $R$, which is defined as $\vec{a}=R \vec{u}$. In this equation, $R$ is the rectangular matrix of $(2 m \times n)$ dimension, $2 m$ is the number of rows, and $n$ is the number of columns. The vector $\vec{u}$ is composed of the current changes $\Delta I_{j}$, $j=1, \ldots, n$, of each dipole magnet (horizontal or vertical), and the vector $\vec{a}$ is the difference in BPM readings with respect to the initial (or nominal) BPM readings, in response to the current changes in individual dipoles:

$$
\vec{u}=\left(\begin{array}{l}
\Delta I_{1} \\
\vdots \\
\vdots \\
\vdots \\
\vdots \\
\Delta I_{n}
\end{array}\right), \quad \vec{a}=\left(\begin{array}{l}
\Delta x_{1} \\
\vdots \\
\Delta x_{m} \\
\Delta y_{1} \\
\vdots \\
\Delta y_{m}
\end{array}\right) .
$$

The response matrix elements $R_{i j}$ can be determined from $R_{i j}=\frac{a_{i}}{u_{j}}, i=1, \ldots, 2 m, j=1, \ldots, n$. The response

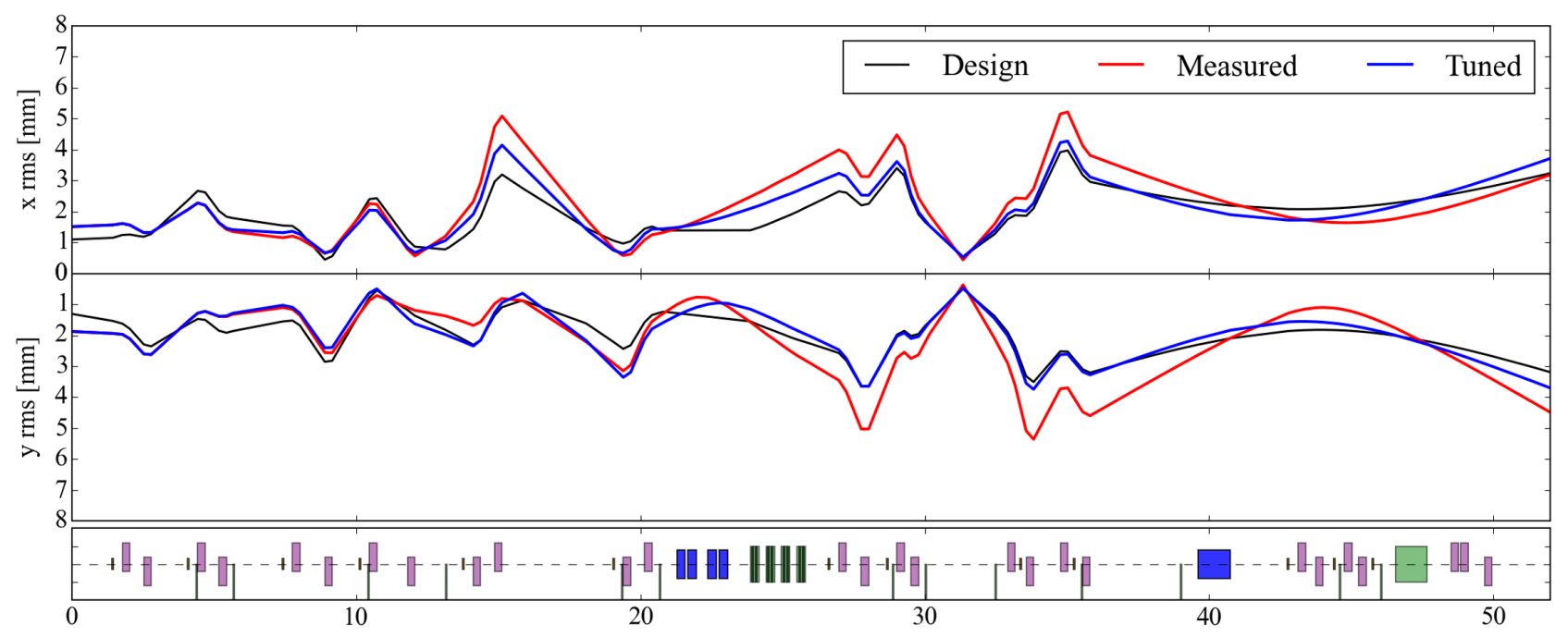

FIG. 4. Argon beam rms envelopes calculated by the FLAME code in the transport section of LS1. 
TABLE I. Argon beam normalized rms emittances at 0.5 and $20.3 \mathrm{MeV} / \mathrm{u}$.

\begin{tabular}{lcccc}
\hline \hline Location & \multicolumn{2}{c}{ MEBT, } & \multicolumn{2}{c}{ After LS1, } \\
\hline $\begin{array}{l}\text { rms emittance } \\
(\pi \mathrm{mm} \mathrm{mrad})\end{array}$ & $\epsilon_{x}$ & $\epsilon_{y}$ & $\epsilon_{x}$ & $\epsilon_{y}$ \\
$\mathrm{I}_{\text {peak }}=33 \mu \mathrm{A}$ & 0.14 & 0.10 & 0.15 & 0.12 \\
$\mathrm{I}_{\text {peak }}=133 \mu \mathrm{A}$ & 0.13 & 0.11 & 0.12 & 0.14 \\
\hline \hline
\end{tabular}

matrix can be used to find the dipole current settings to align the beam such that the BPM readings will be close to zero. The required change in the setting of dipole currents can be found from this linear algebraic equation:

$$
\vec{u}=R^{+} \vec{a},
$$

where $R^{+}=\left(R^{T} R\right)^{-1} R^{T}$ is the pseudoinverse matrix [8]. In practice, Eq. (1) does not provide an immediate solution to achieve zero readings from BPMs, meaning that the beam central trajectory is fully corrected. Therefore, the steering correction was performed in an iterative manner: First, we requested the magnet current settings which reduce the beam centroids' deviations (BPM readings) by a factor of 2 . The same procedure was applied again to reduce beam centroid deviations in the BPMs by another factor of 2 , etc.
The beam steering correction was considered complete when the BPM readings were within the accuracy of the BPM alignment, which is typically within $\pm 0.5 \mathrm{~mm}$. The ORM-based application works faster if it is applied to smaller sections of LS1, containing 5-10 steering dipole magnets. The results of beam steering correction at LS1 are shown in Fig. 5.

\section{B. High-power equivalent beam}

After fine-tuning and optimizing both longitudinal and transverse dynamics of the argon beam, another three ion species $\left({ }^{20} \mathrm{Ne}^{6+},{ }^{86} \mathrm{Kr}^{17+}\right.$, and $\left.{ }^{129} \mathrm{Xe}^{26+}\right)$ were accelerated to a beam energy of $20.3 \mathrm{MeV} / \mathrm{u}$ by simply scaling all electromagnetic fields with respect to the established ${ }^{40} \mathrm{Ar}^{9+}$ tune with appropriate charge-to-mass ratios. The transmission through LS1 was $100 \%$ at $\sim 1.5 \%$ calibration and measurement accuracy of BCMs. For accurate beam current measurements, BCM readings were averaged over several minutes due to beam current reading fluctuations resulting from signal cross talk from nearby ion vacuum pumps. Near the start of the beam commissioning in LS1, we observed $\sim 2 \times 10^{-4}$ level relative beam losses in the HMRs after the first three cryomodules. Final matching of the MEBT beam to the linac reduced the beam losses on HMRs to the baseline noise level. The tuning and setting of electromagnetic fields in all accelerator devices were

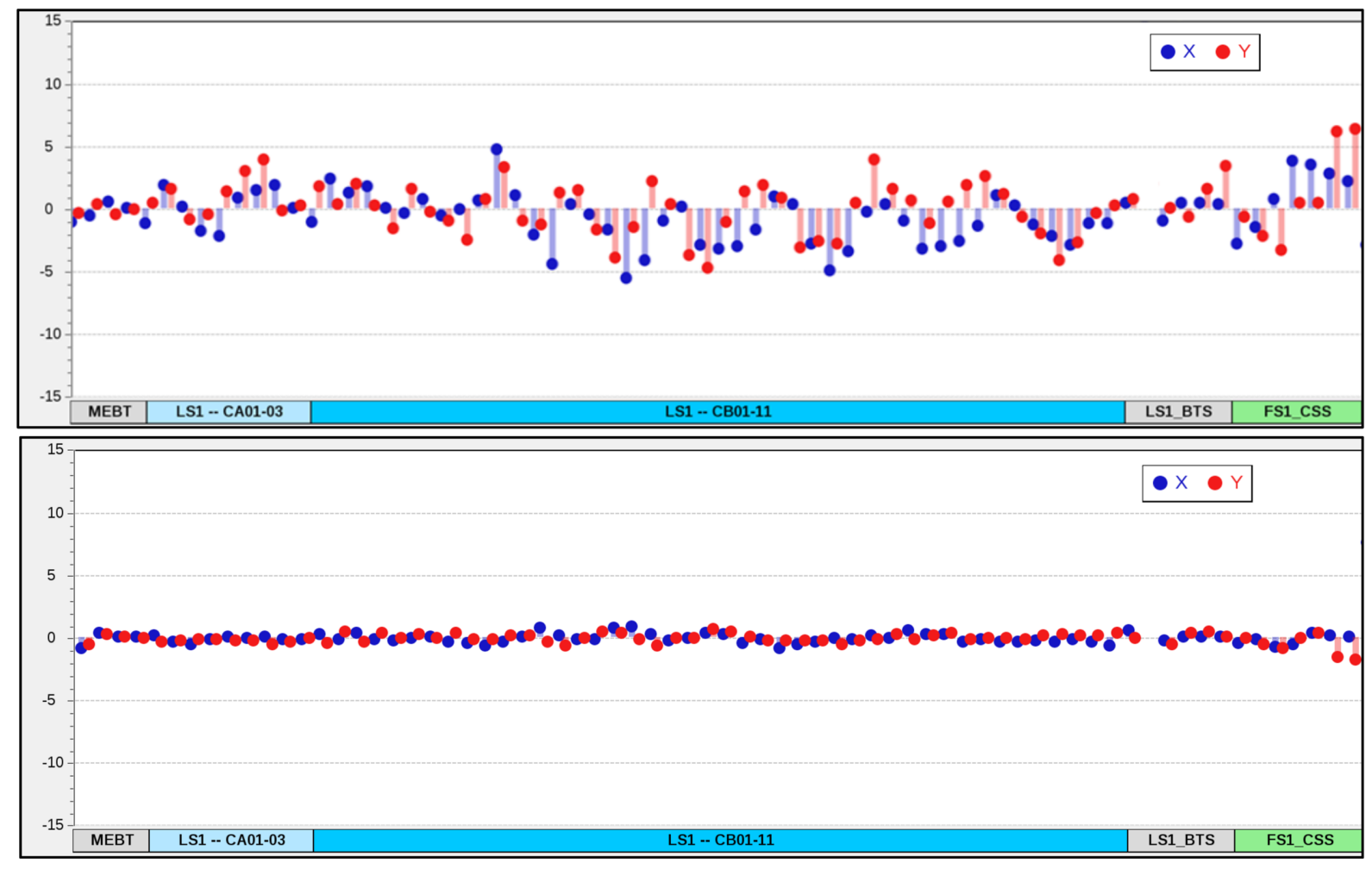

FIG. 5. BPM readings along LS1 prior to (top) and after the application of an ORM-based beam steering correction (bottom). The vertical scale is in millimeters, and the horizontal length is $120 \mathrm{~m}$ and includes MEBT, LS1, and the stripper. 


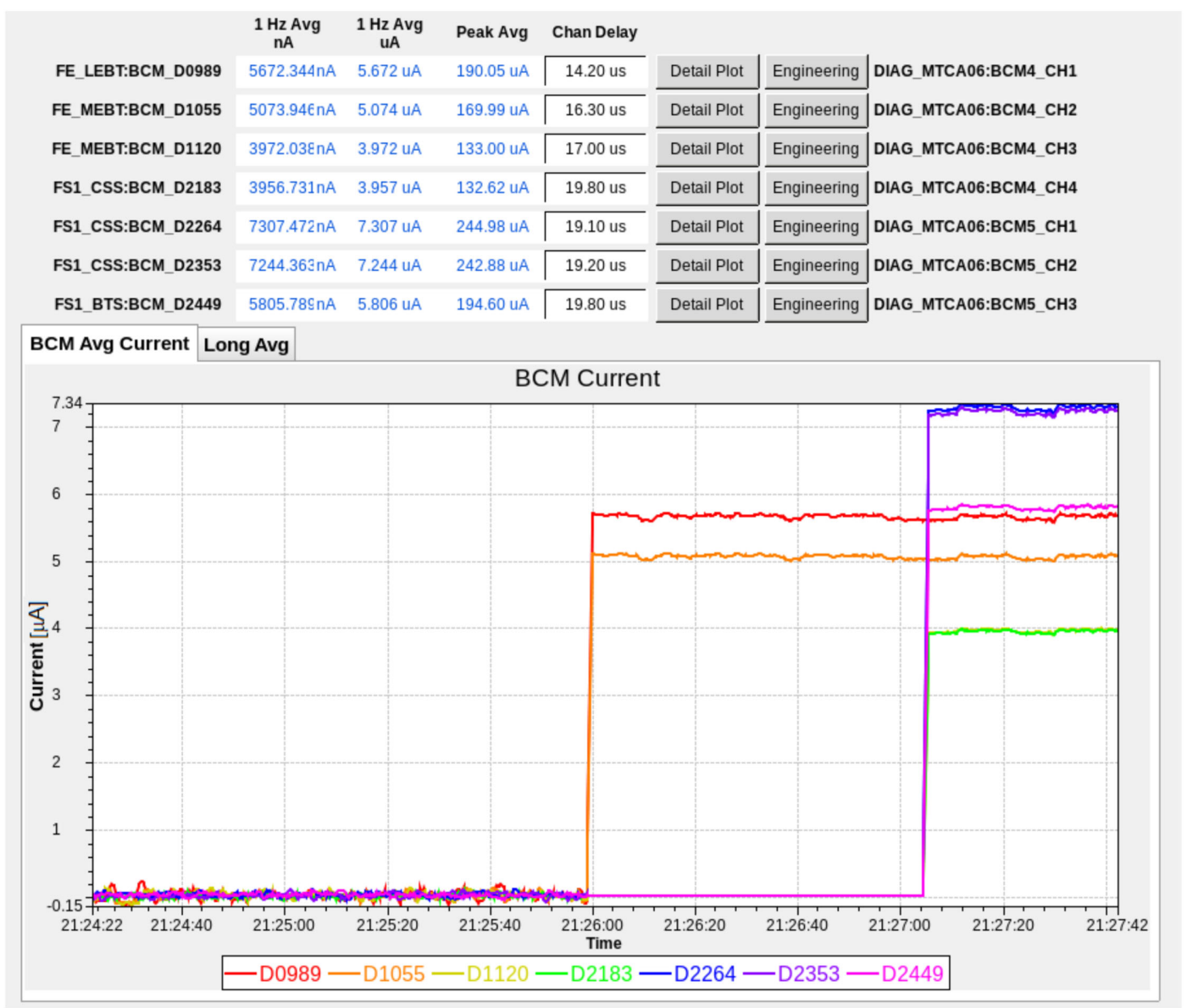

FIG. 6. Screenshot of BCM currents during the acceleration of a $133 \mu \mathrm{A}$ argon beam in LS1. The locations of BCMs are D0989, upstream of the RFQ; D1055, at the exit of the RFQ; D1120, upstream of the first cryomodule; D2183, upstream of the stripper; D2264, downstream of the stripper; D2353, upstream of the $45^{\circ}$ bending magnet; and D2449, upstream of FS1b.

performed with a very low beam power, typically below $2 \mathrm{~W}$, for both the transverse and longitudinal tuning. The acceleration of the high-power equivalent beam was demonstrated in two regimes: (i) high peak current, low duty factor and (ii) low current cw beams. During this commissioning stage, the average beam power accelerated in LS1 was limited by the beam dumps to $500 \mathrm{~W}$. Charge stripping is required to reduce the beam rigidity to deliver it to FS1b. During the beam commissioning campaign, the high-power equivalent beam to the FS1b beam dump was transported in five different modes, shown in Table II. Each mode was operated briefly, for about one minute, in order to minimize the activation of the beam dump shielding. The highest peak current was $14.8 \mathrm{p} \mu \mathrm{A}$ of the argon beam, which corresponds to $30 \%$ of the FRIB ultimate design intensity. The screenshot of BCM measurements in various locations along LS1 is shown in Fig. 6. The charge selector slits absorb $10 \%$ of the argon beam power in charge states $16+$ and $17+$. Three charge states of the krypton beam $(33+, 34+$, and $35+)$ were transported to the beam dump, and only $3 \%$ of the total power in charge states $32+$ and $36+$ was absorbed by the charge selector slits.

TABLE II. High-power equivalent beam modes into FS1b.

\begin{tabular}{|c|c|c|c|c|c|c|c|}
\hline Mode & $\begin{array}{c}\text { Ion } \\
\text { species }\end{array}$ & $\begin{array}{c}\mathrm{I}_{\text {peak }} \text { in LS1 } \\
(\mu \mathrm{A})\end{array}$ & $\begin{array}{c}\text { Pulse } \\
\text { length }(\mu \mathrm{s})\end{array}$ & $\begin{array}{l}\text { Repetition } \\
\text { rate }(\mathrm{Hz})\end{array}$ & $\begin{array}{c}\text { Duty } \\
\text { factor }(\%)\end{array}$ & $\begin{array}{l}\text { Average beam } \\
\text { power at charge } \\
\text { selector }(\mathrm{W})\end{array}$ & $\begin{array}{c}\text { Average beam } \\
\text { power in beam } \\
\text { dump } 2(\mathrm{~W})\end{array}$ \\
\hline 1 & $\mathrm{Ar}$ & 34.3 & 1000 & 100 & 10 & 31 & 278 \\
\hline 2 & $\mathrm{Ar}$ & 3.2 & 9995 & 100 & $\mathrm{cW}$ & 29 & 260 \\
\hline 3 & $\mathrm{Ar}$ & 133 & 300 & 100 & 3 & 36 & 324 \\
\hline 4 & $\mathrm{Ar}$ & 133 & 6000 & 5 & 3 & 36 & 324 \\
\hline 5 & $\mathrm{Kr}$ & 13.7 & 1000 & 100 & 10 & 3 & 138 \\
\hline
\end{tabular}




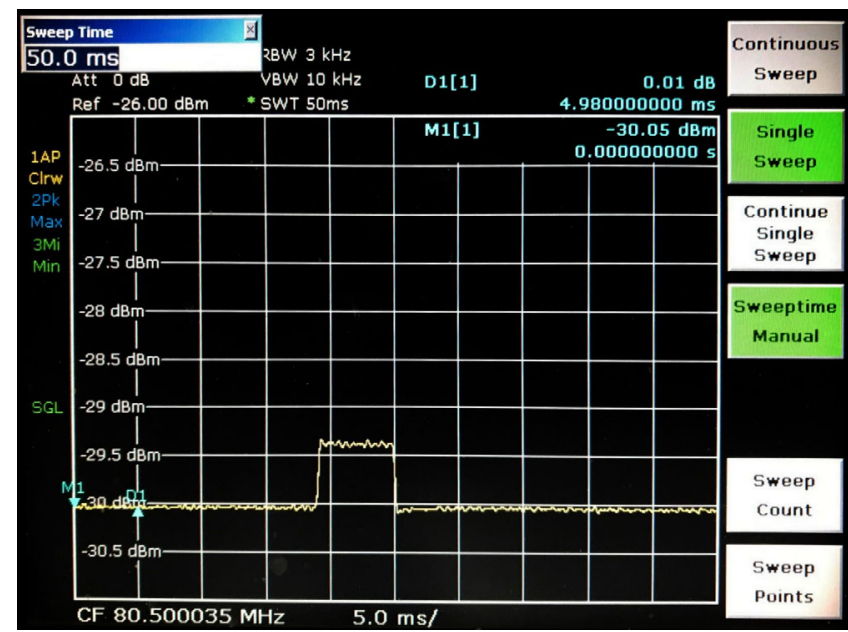

FIG. 7. Oscilloscope screenshot showing the forward beam power increase to compensate beam loading. The horizontal axis is the time with $5 \mathrm{~ms}$ per division, and the vertical axis is the forward $\mathrm{rf}$ power; $-30 \mathrm{dBm}$ represents $320 \mathrm{~W}$ forward $\mathrm{rf}$ power.

\section{Beam loading}

One of the methods to increase the average beam power in the FRIB linac is to increase the beam duty factor after the completion of fine-tuning with the high peak current. A proper compensation of the beam loading effects in the SC cavities is required to keep the beam dynamics and possible beam losses independent of the beam pulse length. The low-level rf (LLRF) feedback control was designed to compensate for beam loading by properly regulating the forward rf amplitude and phase $[9,10]$. Beam mode 4 , as shown in Table II, was specifically used to test the LLRF feedback control for the beam loading compensation in all SC cavities in LS1. The beam pulse length $(6 \mathrm{~ms})$ was comparable to the cavity filling time of $8 \mathrm{~ms}$. Figure 7 shows an oscilloscope screenshot of the forward rf power to one of the $\beta_{\text {opt }}=0.085$ cavities increasing by $16 \%$ to compensate the transient beam-loaded voltage. The forward rf phase, measured by the LLRF controller, also changed by approximately $3^{\circ}$. As a result, the cavity field amplitude and phase were kept almost constant with changes due to the beam loading effect less than $\pm 0.1 \%$ and $\pm 0.1^{\circ}$, respectively.

\section{BEAM STRIPPING}

The FRIB driver linac will include a liquid lithium stripper, as shown in Fig. 1, to boost the charge state of all ion beams at energies from 17 to $20 \mathrm{MeV} / \mathrm{u}$, depending on the ion species [11]. Since the lithium stripper is not yet available in the tunnel, thin carbon foils were utilized for charge stripping of $20.3 \mathrm{MeV} / \mathrm{u} \mathrm{Ne}, \mathrm{Ar}, \mathrm{Kr}$, and $\mathrm{Xe}$ ion beams in our experiments. By fitting with the FLAME code [2], a very small beam size, with a $0.5 \mathrm{~mm}$ rms radius, can be created on the stripping foil as shown in Fig. 8 . A magnetic quadrupole quadruplet was used to change the beam rms size on the foil. A second quadruplet, downstream of the charge stripper, was used to restore beam matching with the following section of the beam transport system.

Figure 9 shows a cw argon beam image on the carbon foil with a thickness of $0.8 \mathrm{mg} / \mathrm{cm}^{2}$. The beam was accelerated in mode 2, shown in Table II, and focused to a $0.5 \mathrm{~mm}$ rms radius on the foil. The carbon foil located to the left in Fig. 9 also had a thickness of $0.8 \mathrm{mg} / \mathrm{cm}^{2}$ and was previously utilized during the acceleration of the beam in mode 3. Significant distortions of the foil can be seen as a result of just $60 \mathrm{sec}$ of beam operation in mode 3. After the high-power operation, we observed a reduction of argon beam intensity in the $18+$ charge state from $92 \%$ to $80 \%$,

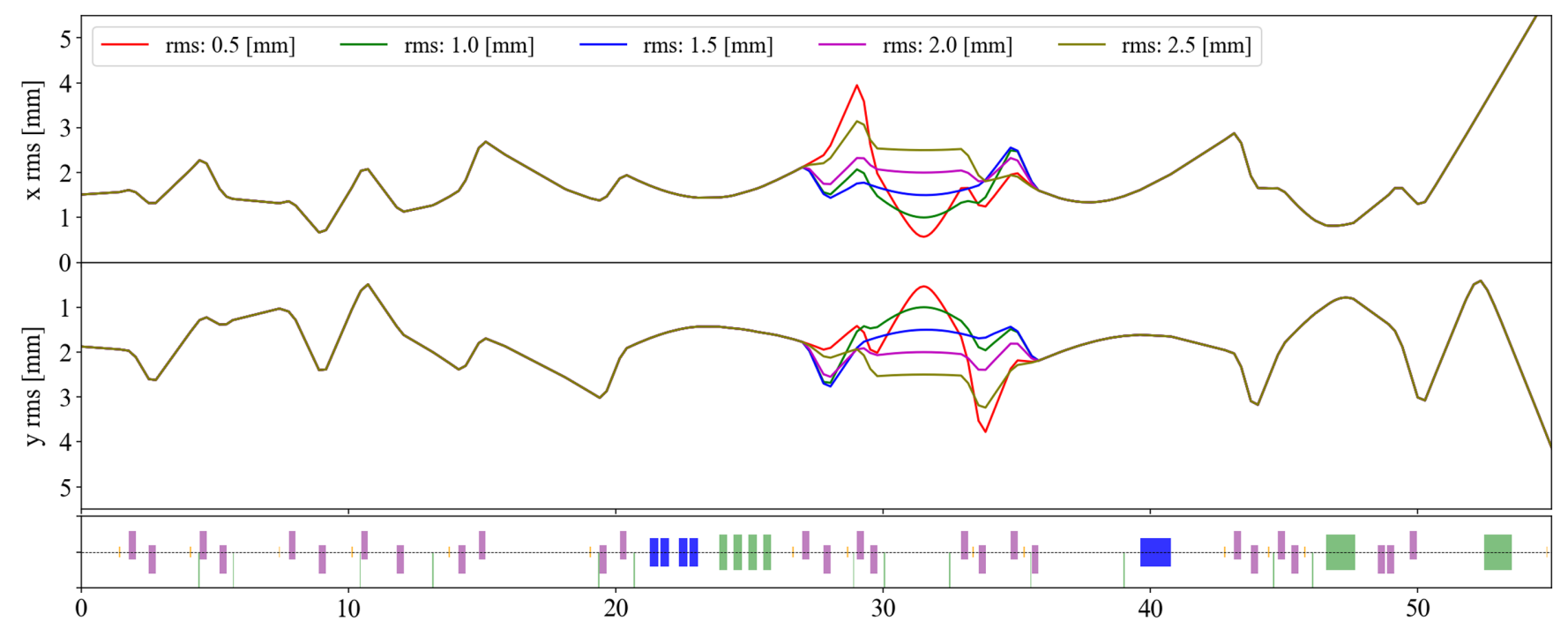

FIG. 8. Argon rms beam envelopes downstream of the accelerator section of the linac. The numbers in the legend indicate the rms beam size at the charge stripper location. 


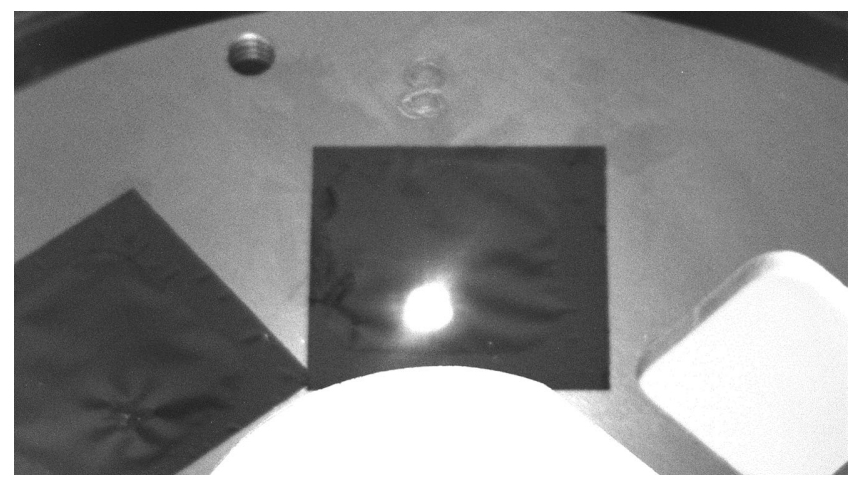

FIG. 9. cw argon beam image on carbon stripper foil. The beam rms and full diameters were $1 \mathrm{~mm}$ and $\sim 3 \mathrm{~mm}$, respectively.

which was caused by the foil thinning during this $60-\mathrm{sec}$ beam interaction with the foil. Clearly, the beam density on a carbon foil should be reduced to extend the lifetime of the stripping foil.

The charge state distributions for four ion species $(\mathrm{Ne}, \mathrm{Ar}$, $\mathrm{Kr}$, and $\mathrm{Xe}$ ) were measured after the $45^{\circ}$ bend, and Fig. 10 summarizes the results. A comparison of the measured data with the calculation using the Baron formula [12] shows that the equilibrium charge state distribution (CSD) was reached with foil thicknesses of 0.4 and $0.8 \mathrm{mg} / \mathrm{cm}^{2}$ for neon and argon beams, respectively. The thickest foil we had available was $0.8 \mathrm{mg} / \mathrm{cm}^{2}$, and this is not sufficient to reach equilibrium CSD for heavier ions such as krypton and xenon.

A neon beam was utilized to study transverse rms emittance growth as a function of both the beam size on the stripper and the stripping foil thickness. For the rms emittance measurements, the quadrupole scan method combined with the profile monitor located upstream of the bending magnet was applied. The neon beam is the most suitable for these measurements, due to stripping into a single charge state. The rms emittance growth factor as a function of both the foil thickness and the beam size on the stripper is shown in Fig. 11. The carbon stripper for the beam commissioning in the FRIB linac will be utilized until the lithium stripper is incorporated into the beam line by the end of 2020. In future experiments, we plan to maintain a $1.5 \mathrm{~mm} \mathrm{rms}$ beam radius on the carbon stripper to increase the foil lifetime. It is likely that the carbon stripper on a rotating wheel will be used as a backup option at FRIB to deliver low-power (up to $\sim 10 \mathrm{~kW}$ ) beams to the target.

\section{DEMONSTRATION OF TWO-CHARGE STATE ION BEAM ACCELERATION}

LS1 is designed to simultaneously accelerate two charge states of uranium $\left(\mathrm{U}^{33+}\right.$ and $\left.\mathrm{U}^{34+}\right)$ in order to meet the beam-power requirement of $400 \mathrm{~kW}$. When a dual charge state beam is accelerated, there are two major effects [13]
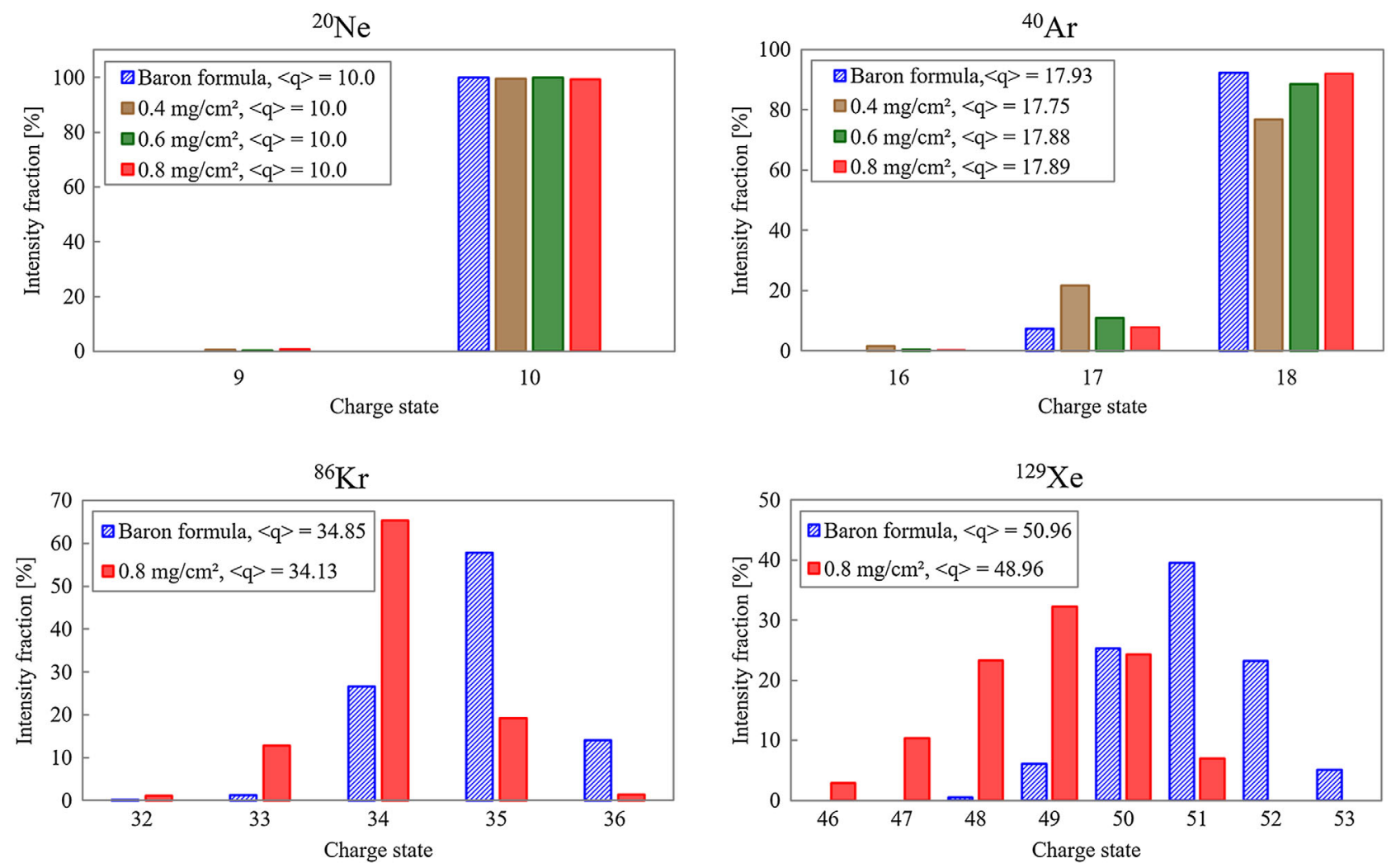

FIG. 10. Charge state distributions for $\mathrm{Ne}, \mathrm{Ar}, \mathrm{Kr}$, and $\mathrm{Xe}$ beams. The stripping foil thickness was not sufficient to reach equilibrium $\mathrm{CSD}$ for $\mathrm{Kr}$ and $\mathrm{Xe}$. 


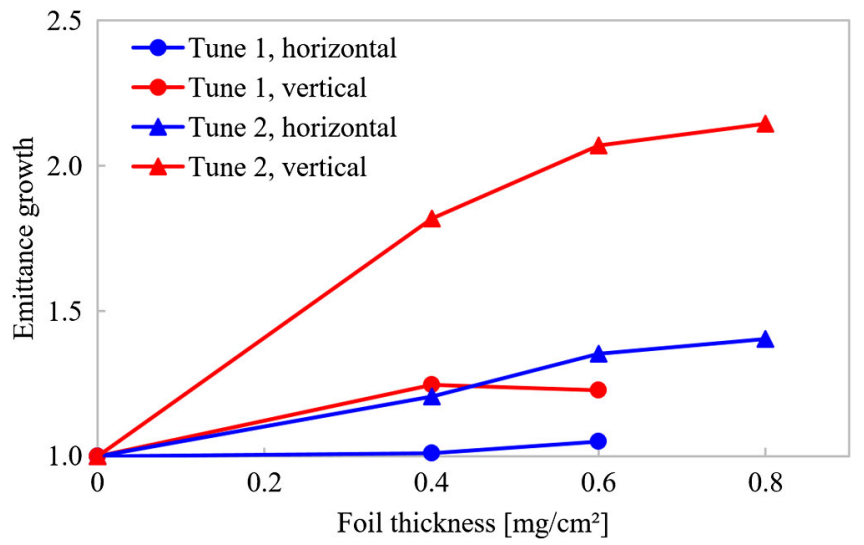

FIG. 11. The rms emittance growth factor as a function of the foil thickness. Tune 1 corresponds to $\mathrm{x}_{\mathrm{rms}}=1.4 \mathrm{~mm}$ and $\mathrm{y}_{\mathrm{rms}}=1.5 \mathrm{~mm}$. Tune 2 corresponds to $\mathrm{x}_{\mathrm{rms}}=2.7 \mathrm{~mm}$ and $\mathrm{y}_{\mathrm{rms}}=4.3 \mathrm{~mm}$.

contributing to the effective emittance growth: (a) the synchronous phase is different for each charge state and (b) misalignments of beam optics elements and the dipole component of the electromagnetic fields in accelerating cavities. The contribution due to these effects is proportional to $2\left(q_{1}-q_{2}\right) /\left(q_{1}+q_{2}\right)$, which is equal to $3 \%$ for the dual charge state uranium beam. At this time, metal beams (including uranium) are not yet available from the ion source. Therefore, in order to mock up simultaneous acceleration of a dual charge state beam, krypton beams with charge states $17+$ and $18+$ were independently accelerated with the same LS1 machine settings through the charge stripper and stripped to higher charge states. Since the relative charge state difference for $\mathrm{Kr}$ in LS1 was $5.7 \%$, we expect a larger impact on the beam quality than in the case of a dual charge state uranium beam.

First, $\mathrm{Kr}^{17+}$ was accelerated to $20.3 \mathrm{MeV} / \mathrm{u}$ and tuned for $100 \%$ transmission, with an accurately aligned central beam trajectory along the linac. The acceleration of dual charge state beams is not yet possible in the current configuration of the front end, because the velocity equalizer has not yet been installed upstream of the RFQ. This device is necessary to inject each component of a dual charge state ion beam into the RFQ with equal velocities $[14,15]$. Therefore, the approach was to transport the $\mathrm{Kr}^{18+}$ beam to the entrance of the RFQ with the same velocity as the $\mathrm{Kr}^{17+}$ beam, which was achieved by only changing the total accelerating voltage of the dc injector. The $\mathrm{Kr}^{18+}$ beam was then accelerated in the RFQ and in LS1 with the same machine settings as the $\mathrm{Kr}^{17+}$ beam. The beams were transported to the second beam dump (FS1b). The transmission of the $\mathrm{Kr}^{18+}$ beam through $\mathrm{LS} 1$ was $100 \%$. The $\mathrm{Kr}^{18+}$ beam energy was measured to be $20.264 \mathrm{MeV} / \mathrm{u}$, compared to $20.304 \mathrm{MeV} / \mathrm{u}$ of the $\mathrm{Kr}^{17+}$ beam. The synchronous phase of the $\mathrm{Kr}^{18+}$ beam was shifted by $\sim 5^{\circ}$ with respect to the $\mathrm{Kr}^{17+}$ beam, which results in an oscillation of the $\mathrm{Kr}^{18+}$ beam bunch phase, as shown in Fig. 12.

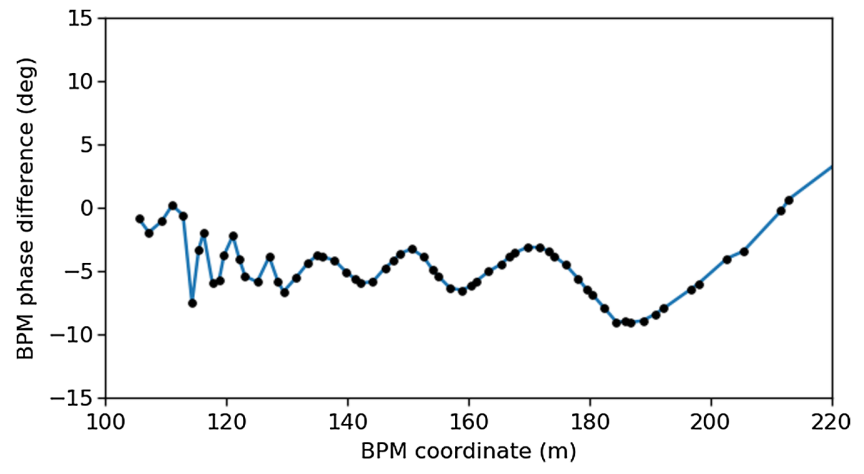

FIG. 12. Deviation of the $\mathrm{Kr}^{18+}$ beam phase in the BPMs along LS1 with respect to the $\mathrm{Kr}^{17+}$.

The LS1 longitudinal tune can be adjusted to minimize the effective longitudinal emittance of any dual charge state ions (heavier than xenon) at the position of the charge stripper, which is located at $223.7 \mathrm{~m}$. The experiments with the acceleration of two charge states of a krypton beam indicate that the low emittance condition can be met. First, the energy difference of krypton beam at charge states $17+$ and $18+$ is small, less than $0.2 \%$. Second, as shown in Fig. 12, the phase difference of krypton beam bunches at charge states $17+$ and $18+$ is close to zero at the location of the charge stripper.

Figure 13 shows the deviation of the $\mathrm{Kr}^{18+}$ beam position with respect to the $\mathrm{Kr}^{17+}$ beam position in all LS1 BPMs. While there is a notable difference in the beam position for krypton charge states $17+$ and $18+$, this difference will be smaller for a dual charge state uranium beam. In addition, with the available number of steering correctors, the central trajectory of a dual charge state beam can be optimized to avoid a large effective emittance growth. Finally, Fig. 14 illustrates profiles of the $\mathrm{Kr}^{34+}$ beam in the high dispersion area obtained with the stripping of either $\mathrm{Kr}^{17+}$ or $\mathrm{Kr}^{18+}$ beams. The profiles overlap well, which is one of the necessary conditions for low emittance growth during the acceleration of a dual charge state beam.

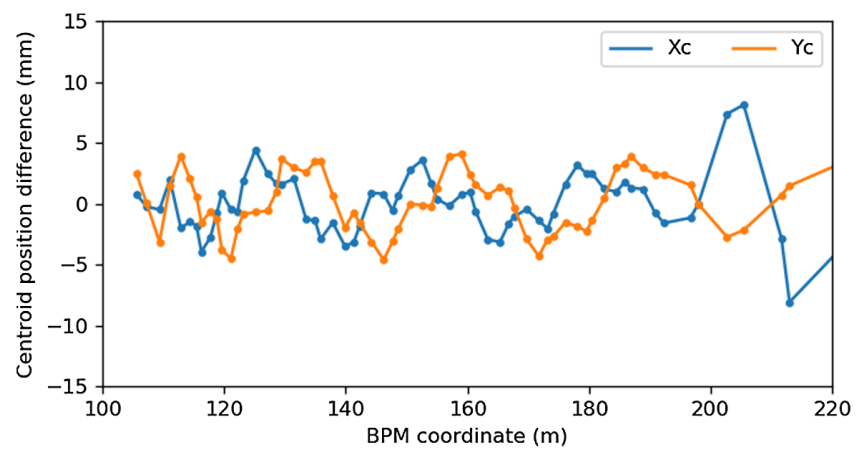

FIG. 13. Deviation of the $\mathrm{Kr}^{18+}$ beam positions in the BPMs along LS1 with respect to the $\mathrm{Kr}^{17+}$ in the horizontal and vertical planes. 


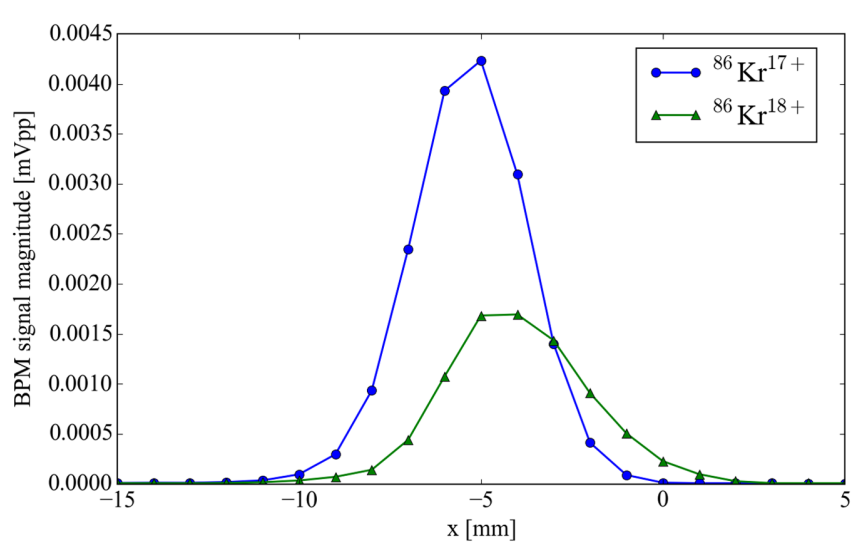

FIG. 14. Horizontal profiles of the $\mathrm{Kr}^{34+}$ beam obtained from $\mathrm{Kr}^{17+}$ and $\mathrm{Kr}^{18+}$ beams at the location of the charge selector in FS1.

These experiments confirm that a dual charge state uranium beam can be successfully accelerated in LS1 in order to nearly double the available power on the target.

\section{SUMMARY}

The first segment of the FRIB linac, composed of 15 cryomodules, was successfully commissioned with a beam. A detailed study of the beam parameters demonstrated good consistency with the original design parameters. Four ion beam species ( $\mathrm{Ne}, \mathrm{Ar}, \mathrm{Kr}$, and $\mathrm{Xe}$ ) were accelerated up to $20.3 \mathrm{MeV} / \mathrm{u}$ with $100 \%$ transmission and no detectable beam losses. The high quality of the accelerated beam was achieved by fine-tuning of both transverse and longitudinal dynamics and successful implementation of a beam central trajectory correction by the optics response matrix method. A high-power equivalent argon beam was accelerated at 3\% duty cycle, with the peak current intensity equal to $30 \%$ of the ultimate design intensity for FRIB. The average beam power was limited at this stage by the beam dump power rating. Beam charge stripping was studied utilizing thin carbon foils. Charge state distributions for all four ion species were measured and compared to the calculations with the Baron formula. It was found that for the heavier ions, such as krypton and xenon, the foil thickness should be greater than $0.8 \mathrm{mg} / \mathrm{cm}^{2}$ to reach equilibrium charge state distribution. The beam loading compensation with the LLRF feedback system was able to successfully maintain the accelerating field within $\pm 0.1 \%$ and $\pm 0.1^{\circ}$ for the amplitude and phase, respectively. Empirical validation of dual charge state acceleration in LS1 was demonstrated with a krypton beam at charge states $17+$ and $18+. \mathrm{Kr}^{17+}$ and $\mathrm{Kr}^{18+}$ beams were independently accelerated, utilizing the same LS1 machine settings, through the charge stripper and stripped to higher charge states. All accelerator hardware showed very reliable operation within the design parameter space.
Currently, the installation of 12 cryomodules of $\beta_{\text {opt }}=$ 0.29 cavities and 12 cryomodules of $\beta_{\text {opt }}=0.53$ at linac segment 2 (see Fig. 1) is in progress. The commissioning of LS2 will take place in March-June 2020 with the goal to accelerate an argon beam above $200 \mathrm{MeV} / \mathrm{u}$.

\section{ACKNOWLEDGMENTS}

The authors greatly appreciate the contributions of all FRIB staff to the successful operation of the FRIB linac. This work is supported by the U.S. Department of Energy Office of Science under Cooperative Agreement No. DESC0000661, the State of Michigan, and Michigan State University.

[1] J. Wei et al., The FRIB superconducting linac-Status and plans, Int. J. Mod. Phys. E 28, 1930003 (2019).

[2] Z. He, Y. Zhang, J. Wei, Z. Liu, and R. M. Talman, Linear envelope model for multi-charge state linac, Phys. Rev. Accel. Beams 17, 034001 (2014).

[3] P. N. Ostroumov, S. Cogan, K. Fukushima, S. Lidia, T. Maruta, A. S. Plastun, J. Wei, J. Wong, T. Yoshimoto, and Q. Zhao, Heavy ion beam acceleration in the first three cryomodules at the Facility for Rare Isotope Beams at Michigan State University, Phys. Rev. Accel. Beams 22, 040101 (2019).

[4] P. N. Ostroumov and K. W. Shepard, Correction of beamsteering effects in low-velocity superconducting quarterwave cavities, Phys. Rev. Accel. Beams 4, 110101 (2001).

[5] P. N. Ostroumov et al., A new generation of superconducting solenoids for heavy-ion linac application, in Proceedings of LINAC'02, Pohang, Korea, p. 332, http://accelconf .web.cern.ch/AccelConf/102/PAPERS/TU413.PDF.

[6] K. Hosoyama et al., Superconducting solenoid package prototyping for FRIB SRF linac, in Proceedings of IPAC'15, Richmond, VA, USA, p. 2886; http://accelconf .web.cern.ch/AccelConf/IPAC2015/papers/wepma051.pdf.

[7] M. G. Minty and F. Zimmermann, Measurement and Control of Charged Particle Beams (Springer, New York, 2003).

[8] G. R. Lindfield and J.E.T. Penny, Numerical Methods, third ed. (Academic, New York, 2012).

[9] J. Vincent, D. Morris, N. Usher, Z. Gao, S. Zhao, A. Nicoletti, and Q. Zheng, On active disturbance rejection based control design for superconducting RF cavities, Nucl. Instrum. Methods Phys. Res., Sect. A 643, 11 (2011).

[10] D. G. Morris et al., RF system for FRIB accelerator, in Proceedings of IPAC'18, Vancouver, Canada, p. 1765, http://accelconf.web.cern.ch/AccelConf/ipac2018/papers/ wexgbf3.pdf.

[11] J. Nolen and F. Marti, Charge strippers of heavy ions for high intensity accelerators, Rev. Accel. Sci. Techol. 06, 221 (2013).

[12] E. Baron, M. Bajard, and Ch. Ricaud, Charge exchange of very heavy ions in carbon foils and in the residual gas of GANIL cyclotrons, Nucl. Instrum. Methods Phys. Res., Sect. A 328, 177 (1993). 
[13] P. N. Ostroumov and K. W. Shepard, Multiple-charge beam dynamics in an ion linac, Phys. Rev. Accel. Beams 3, 030101 (2000).

[14] P. N. Ostroumov et al., Heavy-ion beam acceleration of two-charge states from and ECR ion source, in Proceedings of the 20th International Linac Conference, LINAC-2000, Monterey, CA, 2000 (SLAC, Menlo Park,
CA, 2000), http://accelconf.web.cern.ch/AccelConf/100/ papers/MOD01.pdf.

[15] Q. Zhao et al., FRIB accelerator beam dynamics design and challenges, in Proceedings of HB'12, Beijing, China, p. 404, https://accelconf.web.cern.ch/accelconf/ HB2012/papers/weo3b01.pdf. 